\title{
11 Flucht und Asyl
}

Dieses Kapitel wurde bei Erstveröffentlichung ohne die korrekte Creative Commons Lizenz veröffentlicht. Die korrekte Lizenz finden Sie am Ende des Kapitels.

Ein Erratum zu diesem Kapitel ist verfügbar unter DOI 10.1007/978-3-658-12533-2_14

(C) SINUS Markt- und Sozialforschung GmbH 2016

M. Calmbach et al., Wie ticken Jugendliche 2016?,

DOI 10.1007/978-3-658-12533-2_11 
Zum Zeitpunkt der Datenerhebung (Sommer 2015) waren Flüchtlinge bereits über Wochen das zentrale Thema in den Nachrichten. Wir haben die Jugendlichen gefragt, was sie von diesem Thema mitbekommen, wie sie zur Thematik stehen, ob sie Kontakt zu Geflüchteten haben und in welcher Rolle sie Deutschland bei der Bewältigung der Herausforderungen der Fluchtmigration sehen.

\section{Das Thema Flucht und Asyl beschäftigt die Jugendlichen und polarisiert.}

Über alle demografischen Gruppen und Lebenswelten hinweg (also nicht nur in den bildungsnäheren Segmenten) sind die Jugendlichen an dem Thema Flüchtlinge interessiert. Dabei lassen sich zwei Lager unterscheiden: Jene Jugendliche, die die weitere Aufnahme von Geflüchteten im Allgemeinen befürworten (die deutliche Mehrheit) und jene, die (mehr oder weniger rigoros) fordern, dass die Zuwanderung begrenzt wird bzw. Leistungen für Asylsuchende eingestellt werden.

Nicht unerwartet dominiert das Flüchtlingsthema auch die Wahrnehmung der aktuellen politischen Situation in Deutschland. Bei den Antworten auf die Frage, was derzeit in unserem Land gut oder weniger gut läuft, zeigen sich klar die unterschiedlichen Einstellungen zu den Neuzuwanderern.

\section{In allen Lebenswelten ist man mehrheitlich für die Aufnahme von Geflüchteten und Asylsuchenden - solange Deutschland die Kapazitäten dafür hat.}

Insbesondere die Jugendlichen der bildungsnahen postmodernen Lebenswelten zeigen sich empathisch und mitfühlend gegenüber den Menschen, die aufgrund der Zustände in ihren Herkunftsländern ihre Heimat verlassen haben. Migration wird als unverschuldete und folgerichtige Konsequenz aus politischen Krisen und Kriegen in den entsprechenden Regionen verstanden. Die Entscheidung zur Flucht und der Mut für einen Neuanfang in einem fremden Land werden teilweise auch 


\section{Assoziationen zu den Begriffen „Nation / Nationalität"}

\section{Verschiedenheit / Gleichheit sprache}

Staatsangehörigkeit

Deutschland

\section{Herkunft}

\section{Patriotismus}

Urlaub

Identität

Vorurteile

Vielfalt

Traditionen

Nationalstolz

Zugehörigkeit

Eigenes Herkunftsland

Land / Länder Abgrenzung

$\begin{array}{ll}\text { Zugehörigkeit } \quad \text { Eigenes Herkunftsland } & \text { Nultureller Austausch } \\ \text { Museen, Denkmäler, Gedenkstätten }\end{array}$

bewundert. Das Recht auf Asyl in Deutschland wird von diesen Jugendlichen nicht in Frage gestellt, vielmehr kritisieren sie jene, die sich trotz der vergleichsweise komfortablen Zustände in Deutschland und Europa nicht solidarisch zeigen. Sie appellieren an die humanitäre Verantwortung eines politisch stabilen und wohlhabenden Landes wie Deutschland, aber auch der europäischen Nachbarn. Besonders empathisch zeigen sich die Sozialökologischen, Expeditiven und Experimentalistischen Hedonisten.

( Ich finde es total gerechtfertigt, dass die hierher kommen, weil jeder Mensch hat das Recht, irgendwo aufgenommen zu werden, wenn sein Leben bedroht wird. Das ist vollkommen in Ordnung und die anderen Länder sollten die auch aufnehmen, auch wenn es nur vorübergehend ist. (weiblich, 16 Jahre, Expeditive)
Typische Zitate zur Illustration

[INT: UND WARUM BIST DU DAFÜR, DASS NOCH MEHR FLÜCHTLINGE AUFGENOMMEN WERDEN?] Weil das besser ist für die. (männlich, 14 Jahre, Prekäre) 
(7) Aber ich finde es eigentlich gut zum Teil, nicht, dass da Krieg ist, sondern einfach, dass diese Menschen fliehen können aus diesem Krieg. (weiblich, 14 Jahre, Experimentalistische Hedonisten)

( Ich finde es besser, wenn im Heimatland Krieg ist, dass sie dann nach Europa kommen, dass sie wenigstens versuchen, sich zu retten und dann nochmal einen Neustart beginnen, um was aus ihrem Leben zu machen. (männlich, 17 Jahre, Materialistische Hedonisten)

( Und ich finde auch, dass die Flüchtlinge ja überhaupt nichts dafür können, dass die aus ihrem Land vertrieben werden. Und für die ist es ja egal, ob die in Deutschland oder einem anderen Land ankommen. Die wollen ja überhaupt nur Schutz. (männlich, 16 Jahre, Prekäre)

( Ja, also ich finde schon, dass mehr Flüchtlinge aufgenommen werden sollten. Weil einfach auch die ganzen Leute, die in überfüllten Booten herkommen und dann von der Regierung ...oder ich bin mir gerade nicht sicher, wer das macht. Aber auf die Boote wird ja auch geschossen, dass die untergehen. Das ist der Hammer eigentlich. Das geht überhaupt nicht. (weiblich, 16 Jahre, Sozialökologische)

Die Menschen haben ja auch krass viel durchgemacht. Und sein Heimatland verlassen zu müssen, und seine ganze Familie zu verlieren, da kann ich nicht verstehen, dass man solchen Menschen nicht hilft. (weiblich, 17 Jahre, Adaptiv-Pragmatische)

ICh persönlich sage, dass das vollkommen übertrieben ist, weil irgendwo müssen die Leute ja wieder hin, und man kann sie ja nicht in ihr Land schicken, wo der Krieg ist. (weiblich, 17 Jahre, Adaptiv-Pragmatische)

I Ich finde, jedes Land, das helfen kann, sollte die aufnehmen, die Hilfe brauchen. (männlich, 15 Jahre, Konservativ-Bürgerliche)

( Kein Mensch ist illegal, würde ich sagen. Weil jeder Mensch hat einen Wert und keiner sollte einfach so wie Dreck behandelt werden. (männlich, 16 Jahre, Materialistische Hedonisten)

Fast ebenso häufig wie die hohe Zahl von Flüchtlingen, die nach Europa kommen, wird spontan die Feindlichkeit gegenüber Flüchtlingen von Jugendlichen als Problem genannt. Vor allem in den postmodernen 
Lebenswelten fordert man mehr Toleranz und Engagement von der deutschen Zivilgesellschaft für Menschen auf der Flucht (bessere Unterbringung, stärkere Integrationsbemühungen, deutlicheres Vorgehen gegen rechte Gewalt an Flüchtlingen). Diese Forderungen richten sich sowohl an die Politik als auch an die Bevölkerung, wobei aus den Formulierungen dennoch eine gewisse persönliche Distanz ("'man" , ,denen" , "die“, , diese armen Leute") spricht, die sich mit dem Befund deckt, dass die meisten Jugendlichen wenig bis gar keinen persönlichen Kontakt zu Flüchtlingen haben.

(7) Die Deutschen könnten mehr auf die zugehen, mehr mit den Flüchtlingen reden. Gut, die verstehen die Sprache nicht, aber man kann sich immer irgendwie verständigen oder denen was anbieten. (weiblich, 16 Jahre, Experimentalistische Hedonisten)

( Wenn ich die Leute höre: "Die können kein Deutsch." Ja, das muss man lernen. Wenn ich von jetzt auf gleich in ein anderes Land gehe, kann ich die neue Sprache auch nicht. Man muss es den Flüchtlingen eben beibringen. (weiblich, 16 Jahre, Experimentalistische Hedonisten)

( Klar, es ist natürlich schwierig, man kann nicht alle aus Afrika und so aufnehmen. Aber ich denke, dass Deutschland auf jeden Fall mehr machen könnte. Und gerade, dass ganz viele Leute so negativ denen gegenüber eingestellt sind, dass sie sagen, das sind Sozialschmarotzer oder so. Das finde ich schlimm, wenn Deutsche das machen. Und dass sich auch die Politik da nicht genug für einsetzt. (weiblich, 17 Jahre, Expeditive)

(1) Was ich richtig schlecht finde, es tut mir leid, ist, dass es so viele dumme Einstellungen gegenüber den Flüchtlingen gibt. Das ist saublöd. Jetzt mal ehrlich: Wie dumm kann man eigentlich sein? Diese armen Leute können überhaupt nichts dafür. Die haben keinen Krieg angefangen. Die führen keinen Krieg. Die flüchten einfach nur, weil sie da nicht mehr leben können, weil sie sonst da sterben würden. (weiblich, 16 Jahre, Experimentalistische Hedonisten)

( In Hessen haben die ein Asyllager aufgebaut, und dann wurden sogar die Helfer von dem Aufbau angegriffen, von der NPD. Da muss ich 
schon sagen, dass ist ein Statement wo man halt immer die anderen Länder für ihre rechten Parteien anguckt. Da muss man in Deutschland auch sagen, dass die NPD noch ein Thema ist. (weiblich, 17 Jahre, Adaptiv-Pragmatische)

(7) Es geht uns als Land bzw. uns in Europa so gut. Da sollte es selbstverständlich sein, dass wir anderen Menschen, denen es nicht so gut geht, helfen. Ich glaube, es wird einfach zu wenig Menschen geholfen. Allein die Tatsache, dass es Menschen gibt, denen nicht geholfen wird, denen aber geholfen werden könnte. Das müsste geändert werden. (weiblich, 15 Jahre, Expeditive)

( Die [ANM.: Sozialleistungsbezieher in Deutschland] kriegen umsonst Geld. Und dann beschweren die sich, wenn es ein Euro weniger wird, weil die Flüchtlinge Hilfe brauchen. Das ist lächerlich. Wir sind eines der wirtschaftlich besten Länder der Welt, aber nein, zu uns dürfen die Flüchtlinge nicht. Warum? (weiblich, 16 Jahre, Expeditive)

Im Zuge der im Sommer 2015 rapide angestiegenen Zahlen von Asylbewerbern sind die meisten Jugendlichen (unabhängig von ihrem lebensweltlichen Hintergrund) aber auch der Ansicht, dass Deutschland künftig nicht alle Menschen aufnehmen kann. Diese Situation wird von den Jugendlichen jedoch lebensweltspezifisch unterschiedlich bewertet.

- In den Lebenswelten der gesellschaftlichen Mitte (Konservativ-Bürgerliche und Adaptiv-Pragmatische) blickt man recht nüchtern auf die Situation: „Wenn das Land voll ist, ist es halt voll." Hier hegt man teilweise auch die Hoffnung, dass die Menschen ohnehin möglichst bald nach Beendigung des Konflikts oder nach Ende der jeweiligen Krisensituation wieder in ihr Herkunftsland zurückkehren können das sei für die Flüchtlinge ebenso wie für Deutschland der Idealfall.

Typische

Zitate zur |llustration
( Ja irgendwann ist halt wirklich kein Platz mehr da. Aber bis dahin kann man die schon nehmen. (männlich, 16 Jahre, Adaptiv-Pragmatische)

Nee, also irgendwann ist halt ein Land voll. Da kann man dann auch nichts mehr machen. Ich verstehe jetzt schon, dass sie nicht mehr so viele Immigranten reinlassen wollen. Also jetzt nicht wegen deren 
Hautfarbe oder der Religion, sondern weil es einfach keinen Platz mehr gibt. Da soll halt das Land helfen, das diese Probleme hat. (männlich, 16 Jahre, Adaptiv-Pragmatische)

(7) Also ich finde schon, dass man so viele aufnehmen sollte, wie es nur geht und gerade diese Kriegsflüchtlinge finde ich halt total wichtig. Aber ich finde, man muss trotzdem an einem gewissen Punkt sagen, man kann ja nicht alle reinnehmen. Aber ich finde es natürlich wichtig, dass man so viele, wie es geht, einfach nimmt. (weiblich, 17 Jahre, Adaptiv-Pragmatische)

( Wir können ja nicht jeden aufnehmen, der jetzt ankommt. Das ist ja für uns auch eine Belastung, diese Asylanten aufzunehmen. Aber das nehmen wir in Kauf dafür, dass die in Sicherheit sind. Und wenn das Problem sich wieder aufgelöst hat, können sie wieder in ihr Land zurück. (männlich, 17 Jahre, Konservativ-Bürgerliche)

- In den bildungsnahen Lebenswelten sieht man sich in einem moralischen Dilemma: Man wünscht sich mit Blick auf das Leid der Geflüchteten zu einem anderen Ergebnis als einer Aufnahmebegrenzung zu kommen. Andererseits finden sich in diesen Lebenswelten jedoch auch Stimmen, die eine Begrenzung der Flüchtlingsaufnahme deswegen für richtig halten, weil sonst keine angemessene humanitäre Versorgung und erfolgreiche Integration der bereits aufgenommenen Flüchtlinge möglich sei.

(1)h finde, das ist schwierig, weil wir ein gutes Land sind, das gute Chancen für sie bieten kann, aber wir können auch nicht zu viel bieten, weil sonst können wir den Flüchtlingen, die da sind, quasi nichts mehr bieten. Deswegen finde ich es schon wichtig, dass gut verteilt wird. (weiblich, 17 Jahre, Sozialökologische)

(7) Aber wenn die dann wieder zu viele aufnehmen, dann finde ich es auch irgendwie kritisch, weil das ist ja dann auch kein Verhältnis für einen Menschen, dann da zu leben wie so ein Tier oder so. Haben die doch erst letzte Woche glaube ich erst gesagt, dass noch nie so viele Flüchtlinge aufgenommen worden sind wie dieses Jahr... also dass die keinen Raum mehr dafür haben. (weiblich, 17 Jahre, Sozialökologische) 
(7) Wenn noch mehr Flüchtlinge kommen und wir versuchen würden, die in unsere Gesellschaft mit einzugliedern richtig, das würde eine ziemliche Herausforderung sein. Ich glaube, man würde es auch nicht schaffen können richtig, weil wenn man denen nicht erlaubt zu arbeiten (...). Da müsste man auf jeden Fall noch mal etwas verändern, was aber auch echt schwierig sein wird. Ich weiß nicht, ob man das schaffen kann. (weiblich, 16 Jahre, Expeditive)

\section{Eine Minderheit äußert, teilweise hasserfüllt, fremdenfeindliche Ressentiments.}

Die Jugend in Deutschland ist nicht frei von rechtspopulistischen bzw. ablehnenden, ausgrenzenden oder gar feindlichen Haltungen gegenüber Ausländern bzw. Flüchtlingen. Der Mehrheit von Jugendlichen, die mehr oder weniger empathisch auf das Thema Flucht und Asyl blicken, stehen Jugendliche gegenüber, die sich - manche eher zögerlich, einige besonders drastisch - gegen eine Aufnahme von Flüchtlingen aussprechen. Kennzeichnend sind dabei Ängste vor Überfremdung und einer gravierenden Veränderung vertrauter Strukturen und Lebensbedingungen in Deutschland. Dabei werden teilweise gängige Klischees und Vorurteile ("zunehmende Kriminalität", "Verfall der deutschen Sprache“, ",weniger Ordnung und Sauberkeit") aufgegriffen. Es ergeben sich weder bei der empathischen noch bei der ablehnenden Haltung nennenswerte Unterschiede zwischen ost- und westdeutschen Jugendlichen, wichtiger ist die Milieuzugehörigkeit. Ein lebensweltlicher Schwerpunkt bei der ablehnenden Haltung zeichnet sich bei der jungen gesellschaftlichen Mitte (den Adaptiv-Pragmatischen) und den bildungsfernen Lebenswelten ab (Materialistische Hedonisten, Prekäre).

Typische

Zitate zur Illustration
Ja, also, ich bin eigentlich nicht rassistisch, aber ich finde, dass Schwarze und Türken nichts in unserem Land zu suchen haben. Die haben ein eigenes Land, und wenn die da verschuldet sind oder damit nicht klarkommen, dann ist das deren Problem. Dann müssen die nicht nach Deutschland kommen und uns das Leben schwer machen. (weiblich, 17 Jahre, Materialistische Hedonisten) 
(1) Es reicht erst mal, muss ich sagen. Das ist ein bisschen kacke, weil Deutschland ist nicht mehr so richtig Deutschland, sondern eher so ein Mischmasch. (weiblich, 14 Jahre, Prekäre)

Wie gesagt, irgendwann - an sich wäre das jetzt auch nicht so schlimm - sieht man nur noch Ausländer in der Innenstadt. Das kommt jetzt vielleicht ein bisschen rassistisch rüber, aber irgendwann nervt mich das dann auch. Ich war letztes Wochenende in $\mathrm{H}$. bei Freunden, und da bin ich die Straße runter gelaufen, und da habe ich nur Dunkelhäutige gesehen die ganze Zeit. Ich dachte, ich bin in Afrika! Es sollte schon deutsch bleiben, vor allen Dingen, wenn man sich dann mit den Leuten unterhält, ich kann das schlecht einschätzen, aber ich finde es nicht so gut, wenn man irgendwann nur noch Ausländer sieht. (männlich, 14 Jahre, Adaptiv-Pragmatische)

(7) Was eigentlich gar nicht gut läuft, sind die ganzen Asylanten, die kommen. Das gefällt mir eigentlich gar nicht. Ich meine, wenn sie wirklich aus ihrem Land flüchten wollen, dann sollen sie hierher ziehen und nicht bei Gott und der Welt Asyl suchen. Und man hat ja auch schon gehört, dass sie Terroristen einschleusen. Und wir haben hier in A. jetzt auch schon solche Container. Und ich habe da eigentlich überhaupt keine Lust drauf. Denn da muss ich ja Angst haben, dass hier jede Nacht jemand einbrechen könnte. Oder wenn ich mal alleine zu Hause bin, muss ich ja mit einem Messer unter dem Kopfkissen schlafen. Ich weiß nicht. Das löst in einem voll die Angst aus. (weiblich, 16 Jahre, Materialistische Hedonisten)

Weniger gut sind die Flüchtlinge gerade, weil laut den Nachrichten ja irgendwie jeder Flüchtling alles umsonst bekommt, das finde ich jetzt nicht so gut. (...) Und dass sie irgendwie alles zumüllen, habe ich gehört und dadurch auch die deutsche Sprache aussterben soll, habe ich gehört, ja. (weiblich, 16 Jahre, Adaptiv-Pragmatische)

Hier sind ganz viele Ausländer dazugekommen. Das ist hier alles voll. Und das mag ich nicht so. Weil die einen immer anreden, wenn man nur die Straße entlangläuft. Und das nervt halt schon. (weiblich, 15 Jahre, Materialistische Hedonisten) 
(Das mit den ganzen Flüchtlingen und so, dass das bei vielen Orten spezialisiert ist und nicht komplett verteilt wird. Es kommen Anfang Oktober hier ins Gewerbegebiet 60 junge Männer rein, und da haben viele etwas dagegen. Ich finde das jetzt auch nicht wirklich prickelnd, weil man halt immer Angst hat, dass die dann irgendwie was klauen und so. (männlich, 14 Jahre, Adaptiv-Pragmatische)

Wie lässt sich diese abwehrende Haltung gegenüber Flüchtlingen erklären? Für die fehlende Solidarität und das mangelnde Verständnis für Schutzsuchende spielen Verteilungskämpfe eine Rolle. Man hat den Eindruck, dass der eigene Zugang zu Wohlstand durch Asylsuchende gefährdet wird, dass der eigene Lebensstandard sinken und Annehmlichkeiten des täglichen Lebens verloren gehen könnten. Man fürchtet, dass der Traum, sich durch harte Arbeit einen bescheidenen Wohlstand aufzubauen, aufgrund von "Leistungserschleichern" platzen könnte. Besonders in der Prekären Lebenswelt werden Flüchtlinge als unmittelbare „Konkurrenten" am Arbeits- und Wohnmarkt angesehen. Auffällig ist, dass sich vor allem in dieser Gruppe Vorurteile gegenüber Geflüchteten mit generell ausländerfeindlichen Ressentiments unreflektiert vermischen.

Typische

Zitate zur

|llustration

Na ja, die können auch mal in ein anderes Land gehen, weil wir brauchen ja auch unsere Plätze. Die Leute, die hier wohnen, dass die ihre Wohnhäuser wenigstens behalten können. Nicht dass die an Flüchtlinge weitergegeben werden und wir aussortiert werden. Das könnte passieren. (männlich, 14 Jahre, Prekäre)

Nachteile? Weniger Arbeit, weil es sind viele Deutsche, die sagen, hier pass auf, wir wollen auch Arbeit haben und dann kriegen die Flüchtlinge die Arbeit, dann kriegen die vielleicht mehr Geld. (weiblich, 17 Jahre, Prekäre)

( Also was nicht so gut läuft, ist das mit den Flüchtlingen. Ich weiß nicht, irgendwie werden das immer mehr, immer mehr, immer mehr. Und wir haben ja auch bald irgendwie irgendwann keinen Platz mehr für die ganzen Flüchtlinge. Ich meine, die jetzt Hartz IV bekommen, die kriegen ja auch schon. Manchmal reicht das ja auch schon gar nicht mehr mit dem Geld und alles. Und dann kommen jetzt noch Flüchtlinge, dann 
geben sie für die mehr Geld aus. Dann geben sie für Griechenland wieder mehr Geld aus. Das Geld kriegen wir alles nicht wieder. (weiblich, 17 Jahre, Konservativ-Bürgerliche)

Was ich jetzt zum Beispiel zur Zeit nicht so gut finde sind die Flüchtlinge. Das finde ich jetzt nicht so prickelnd. Weil wir haben ja jetzt auch ein Flüchtlingslager in der Schule und dann nehmen die unsere Turnhalle weg. Ich will ja auch Sport machen in der Schule. (männlich, 15 Jahre, Adaptiv-Pragmatische)

Aber die sollen sich keine Wohnungen suchen, weil tausende Deutsche keine Wohnungen haben oder finden. Also es würde mehr Wohnungen geben für Deutsche, wenn keine Türken hier wohnen würden, weil die Türken nehmen sich ja auch immer die größten Wohnungen. Und das verstehe ich nicht. Also warum? (weiblich, 17 Jahre, Materialistische Hedonisten)

I Ich finde das nicht gut. Die haben jetzt auch den Edeka zugemacht, weil die Flüchtlinge in dem Edeka wohnen müssen. Ich finde das überhaupt nicht gut. Nur weil die jetzt kommen, wird alles zugemacht. Andere müssen darauf verzichten, weil die da wohnen müssen. (weiblich, 15 Jahre, Materialistische Hedonisten)

\section{So genannte Wirtschaftsflüchtlinge werden v. a. in den bürgerlichen und bildungsfernen Lebenswelten als "Bedrohung" für Deutschland gesehen.}

Für Jugendliche aus der gesellschaftlichen Mitte ist bei der Debatte um Flüchtlinge die Unterscheidung zwischen „Wirtschafts"- und Kriegsflüchtlingen wichtig ${ }^{9}$. Erstere haben für sie in Deutschland keinen Platz, und von Kriegsflüchtlingen erwarten sie einen großen Integrationswillen, der ihre Aufnahme in Deutschland rechtfertigen soll. Dass die Geflüchteten die deutsche Sprache erlernen, erachten die Jugendlichen als

9 Der Begriff „Wirtschaftsflüchtling“ impliziert eine spezielle Form des „Asylmissbrauchs“ durch vermeintlich nur vorgeschützte politische Fluchtgründe bei vorrangig wirtschaftlichen und sozialen Migrationsmotiven, so dass eine Suche nach besseren wirtschaftlichen und sozialen Existenzbedingungen als "freiwilliges" Wanderungsmotiv unterstellt wird (vgl. http://www.bpb.de/apuz/207999/zur-karriere-abschaetziger-begriffe-in-der-deutschen-asylpolitik? $\mathrm{p}=$ all). Da sich diese denunziatorische Konnotation auch in einigen Zitaten der Jugendlichen spiegelt, wird der Begriff hier dennoch verwendet. 
grundlegende Voraussetzung für ihre Integration auf dem Arbeitsmarkt. Dies knüpft an den Befund der Vorgängerstudie "Wie ticken Jugendliche 2012?" an: Jugendliche aus der gesellschaftlichen Mitte fürchten am stärksten um die Leistungsfähigkeit und den Wohlstand in Deutschland, weil man in Teilen von geringeren Anpassungs- und Leistungsanstrengungen der Zugewanderten ausgeht.

(1) Also für mich sind Flüchtlinge in dem Sinne akzeptabel, wenn es Kriegsflüchtlinge sind oder auch wegen religiöser Hinsicht. Aber auf gar keinen Fall wegen der finanziellen Lage. Das sind für mich keine Flüchtlinge. Die suchen einfach nur nach einem besseren Leben. (weiblich, 16 Jahre, Konservativ-Bürgerliche)

(7) Es ist eine gute Tat von Deutschland. Es ist schön, dass Menschen aufgenommen werden und denen geholfen wird. Aber es gibt ja auch welche zwischen diesen Flüchtlingen, die eigentlich gar keine Hilfe brauchen und nur hier hinkommen, um Geld zu verdienen. (männlich, 15 Jahre, Konservativ-Bürgerliche)

( Irgendwo ist es ja auch eine Belastung. Die sind ja nicht in der Lage, zu arbeiten oder so. Die können auch meistens die Sprache nicht. Das ist ja an sich nichts Schlimmes, sie sind ja auch nur geflohen, die können ja nichts dafür. (männlich, 14 Jahre, Adaptiv-Pragmatische)

Die Ausgrenzung von Menschen, die aufgrund der wirtschaftlichen Lage in ihrem Herkunftsland flüchten, wird von den Jugendlichen damit begründet, dass sie dem Staat lediglich „auf der Tasche liegen“ und ihm "nichts bringen" - eine Meinung, die sich verstärkt in den unterschichtigen Lebenswelten findet, dort auch unter Jugendlichen mit Migrationshintergrund.

(7) Mehr Flüchtlinge? Ich glaube eher, bis zu einer begrenzten Zahl. Weil desto mehr Flüchtlinge, dann denken die: Okay, Deutschland ist so ein Gebiet, wo alle Flüchtlinge hingehen können. Dann wird es auch immer voller und dann wird es, wie soll ich sagen, dann wird es einfach $z u$ viel. (männlich, 16 Jahre, Migrationshintergrund, Materialistische Hedonisten) 


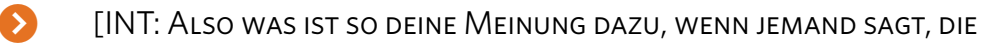
FLÜCHTLINGE KOMMEN NUR HIERHIN UM HERUMZUSCHNORREN?] Na, eigentlich hat der auch recht. Wenn man so guckt, was die Flüchtlinge so machen. Da sollte man ja dafür auch etwas tun und nicht einfach sagen: Ich nehme mal das Geld von den anderen. Weil man sollte auch etwas dazu tun. (männlich, 16 Jahre, Materialistische Hedonisten)

( Auf jeden Fall nicht die ganze Masse, die jetzt gerade auf dem Weg hierher ist, aber schon die, die irgendwie ein Recht darauf haben. Aber die, die nur hierher wollen und das Geld haben wollen und denken, sie können auf der faulen Haut liegen, die können gerne wieder zurück. (weiblich, 17 Jahre, Migrationshintergrund, Materialistische Hedonisten)

Zudem besteht in den Lebenswelten, die noch am ehesten eine Abwehrhaltung gegenüber Flüchtlingen und Ausländern zeigen, oft wenig Neugier und die Bereitschaft, sich auf Fremdes einzulassen. Man ist daher besonders anfällig für Ressentiments. Aus der politischen Sozialisationsforschung ist zudem bekannt, dass Jugendliche erst ab etwa dem 14. Lebensjahr beginnen, sich eigenständig politisch zu interessieren und zu informieren. Viele der in der vorliegenden Studie Befragten haben also womöglich noch keine wirklich informierte politische Meinung ausgebildet. Gerade die Jüngeren - insbesondere aus bildungsbenachteiligten Elternhäusern - sind anfällig für Ressentiments gegenüber Ausländern, weil sie politisch noch recht orientierungslos sind. Es fällt ihnen (noch) schwer, die Thematik abseits von Klischees einzuordnen.

\section{Im Umgang mit Flüchtlingen wird Deutschland im europäischen Vergleich positiv bewertet.}

In der Wahrnehmung vieler Jugendlicher ist Deutschland Vorreiter bei der Aufnahme von Geflüchteten. Man kritisiert in diesem Zusammenhang, dass die anderen europäischen Länder, insbesondere die reicheren und größeren, sich noch nicht stark genug für eine Aufnahme der Flüchtlinge einsetzen. Man fordert eine fairere Verteilung der Geflüchteten, besonders auf die EU-Mitgliedsstaaten. 
Typische Zitate zur |llustration

( Ich denke, Deutschland macht zurzeit sehr viel dafür. Es gibt andere Länder, die gar nicht aufnehmen. Diese Länder sollten jetzt handeln. Ich finde, Deutschland handelt richtig. (männlich, 16 Jahre, Adaptiv-Pragmatische)

( Aber ich fände es zum Beispiel besser, wenn das auch auf verschiedene Länder aufgeteilt wird. Weil der Großteil geht ja nach Italien und nach Deutschland. Ein Teil auch nach Frankreich, aber das war's eigentlich so gut wie. Und das sind ja mehrere Länder der Europäischen Union. Und ich finde, das sollte besser aufgeteilt werden. (männlich, 16 Jahre, Materialistische Hedonisten)

( Aber ich finde es halt schade immer noch wegen den anderen Ländern, dass irgendwelche Länder einfach leer sind und andere voll, voller Menschen. Das sollte ein bisschen ausgeglichener sein. (männlich, 16 Jahre, Adaptiv-Pragmatische)

( ICh finde es eigentlich gut, dass wir die Flüchtlinge aufnehmen. Aber ich finde es von den anderen Ländern scheiße, dass die das nicht machen. (männlich, 17 Jahre, Experimentalistische Hedonisten)

( Obwohl ich Großbritannien auch nicht wirklich gut finde, da ist ja auch noch dieses royale Haus, die Queen, und die sind im Endeffekt auch wieder alle rassistisch. Das war ja mit den Asylanten, wo die ganz klar gesagt haben: Wir nehmen keine Asylanten auf, wir wollen die hier nicht haben. (männlich, 17 Jahre, Konservativ-Bürgerliche)

( Dass sich vielleicht andere Länder hier in der EU dafür einsetzen, dass die auch noch welche aufnehmen. Außer Griechenland sind die ja auch nicht wirklich arm. Das könnte man ändern. Man merkt ja, es wird hier langsam wirklich ganz schön voll. Wenn jetzt schon Turnhallen und alles benutzt werden müssen. Das muss ja nicht sein. (weiblich, 16 Jahre, Experimentalistische Hedonisten)

Ich finde, langsam haben sie genug aufgenommen. Ich finde, die anderen Länder sollten jetzt langsam wirklich aktiv werden. Das machen die hier nicht mehr lange mit, die Bewohner, die dagegen sind. (weiblich, 16 Jahre, Experimentalistische Hedonisten) 
( Das sollten die in den ganzen europäischen Ländern eigentlich verteilen. Nicht dass alle nach Deutschland kommen, weil die deutschen Bürger, die hier wohnen, die haben ja auch ihre Lasten, die müssen ja dann noch mehr Steuergelder bezahlen. (männlich, 15 Jahre, Prekäre)

\section{Jugendliche nehmen beim Thema Integration Staat und Zugewanderte gleichermaßen in die Pflicht.}

Die meisten Befragten formulieren klare Aufgaben hinsichtlich der Bewältigung der anstehenden Integrationsleistungen. Den deutschen Sozialstaat nimmt man in die Verantwortung, den Spracherwerb zu organisieren, Nahrung und Unterkunft sicherzustellen und den Übergang in die Arbeits- bzw. Ausbildungswelt zu ermöglichen. Von den Zugewanderten erwartet man zuvorderst, dass sie bereit sind, schnell und ehrgeizig die deutsche Sprache zu lernen. Einige Jugendliche stellen aber auch klare kulturelle Assimilationsforderungen. Auffällig ist, dass Jugendliche oft eine deutlich von Mitleid geprägte paternalistische Haltung gegenüber Zuwandernden haben.

$>$ Der Staat könnte sie mehr unterstützen mit Hilfsorganisationen, dass sie Deutsch lernen und ins Arbeitsleben rein finden, dass die Firmen Praktika anbieten, extra für Flüchtlinge. (männlich, 15 Jahre, Adaptiv-Pragmatische)

(7) Zum einen, dass sie in Heimen unterkommen können. Und sie könnten ja anfangen, außerhalb der Schule auch nochmal so einen Deutschkurs machen oder so was, dass sie halt besser rein kommen, sage ich jetzt mal so, dass sie auch alles verstehen können. Und ja, halt Anpassung. Also anpassen zu den Deutschen, sage ich jetzt mal. Dass sie halt nicht so auffallen. (männlich, 15 Jahre, Prekäre)

[INT: WAS KÖNNEN DIE LEUTE SELBST MACHEN, UM SICH HIER BESSER EINZULEBEN?] Interessiert daran sein halt. Für sich selber das Ziel haben, ein eigenes Leben aufzubauen, eine eigene Wohnung oder so. Wenn sie es schaffen, einen eigenen Beruf und dass es halt Zeit für Zeit immer größer wird. Dass die hilfsbereit sind, dass die bei den Leuten 
hier mit anfassen, denen helfen, sie hier unterstützen. Im Heimatverein oder so, dass sie da mithelfen oder was mit aufbauen und so. (männlich, 14 Jahre, Adaptiv-Pragmatische)

(7) Dass sie sich eigentlich einfach hier anpassen, weil man sieht jetzt hier dann auch, zum Beispiel in Großstädten, wie zum Beispiel Berlin, sieht man dann auch immer solche Verschleierten, dass sie sich dann einfach anpassen. Weil ich fahre ja auch nicht, wenn ich zum Beispiel nach Indien fahre, würde ich mich ja versuchen, auch anzupassen. Ich würde zwar nicht so lange Schleier-Kleider tragen, aber vielleicht lange Sachen tragen, und das könnten die bei uns auch machen, also anpassen. (männlich, 14 Jahre, Konservativ-Bürgerliche)

Vielleicht vom Staat noch irgendwie mehr Deutschkurse und eben noch irgendwie mehr internationale Treffpunkte, wo sich eben Deutsche und Migranten dann treffen und unterhalten können. Wo eben vielleicht auch ein Dolmetscher da ist, falls man sich noch nicht so verständigen kann. (männlich, 16 Jahre, Adaptiv-Pragmatische)

(Deutschland tut ja eigentlich schon hier einen unterstützen. Es gibt, soweit ich weiß, Kurse, in die die Frauen gehen können, Deutsch lernen können. Meine Mutter war nicht in so einem Kurs, aber kann schon sehr gut Deutsch sprechen. Aber sonst, ich weiß nicht. Vielleicht sollte man die Menschen dazu zwingen, in diesen Kurs zu gehen, bis sie Deutsch können. Einfach aus dem Grund, ich finde es wichtig, dass die Menschen, die hierher kommen, auch Deutsch lernen. Ich kenne viele Personen, die aus einem anderen Land hierher gezogen sind im Nachhinein und die Sprache nicht können. Und ich finde es traurig, wenn eine Mutter nicht mit der Lehrerin von ihrem Sohn sprechen kann. Da sollte Deutschland die Eltern zu zwingen. (weiblich, 16 Jahre, Konservativ-Bürgerliche) 


\section{Einige Jugendliche aus bildungsnahen Elternhäusern erwähnen demografische und wirtschaftliche Vorteile durch Zuwanderung.}

Insbesondere in den bürgerlichen Lebenswelten (bei den Adaptiv-Pragmatischen und Konservativ-Bürgerlichen) begründet man die Offenheit gegenüber Geflüchteten neben einer humanitären Verantwortung Deutschlands auch recht sachlich damit, dass Deutschland bedingt durch die Alterung und Schrumpfung der deutschen Bevölkerung von Asylsuchenden profitieren könnte. Hier steht man daher insbesondere der Zuwanderung von akademisch oder beruflich qualifizierten Menschen offen gegenüber. Diese Meinung wird auch bereits von einigen der jüngeren Befragten geäußert. Auffällig ist dabei, dass diese Jugendlichen alle aus bildungsnahen Elternhäusern kommen.

(1) Man muss auch sehen, dass die Bevölkerungsrate von den Deutschen an sich zurückgeht. Nur durch Einwanderer bleibt die gleich. (weiblich, 17 Jahre, Adaptiv-Pragmatische)

Typische Zitate zur Illustration

( Ich denke schon, dass sie eigentlich mehr aufnehmen könnten, weil es ist ja schon so, dass viele Deutsche gar keine Kinder haben möchten. Und dann, ich weiß nicht, denke ich eigentlich schon, dass wir noch mehr vertragen können. (weiblich, 15 Jahre, Adaptiv-Pragmatische)

( Dass Leute einwandern, finde ich okay, insofern sie irgendetwas vorzuweisen haben. Ich finde nicht, dass wir jeden aufnehmen sollten. Das hat auch nichts mit dem Land, aus dem die Person kommt, zu tun, sondern man sollte immer versuchen, gebildete Leute ins Land zu bekommen. Wir haben ja das Problem, dass wir ganz viele einfache Arbeiter aus der Türkei hergeholt haben, die dann auch zum Großteil geblieben sind. (männlich, 17 Jahre, Konservativ-Bürgerliche)

( Und außerdem ist Deutschland ja generell unterbevölkert. Das kann man ja auch mal dazu sagen. Ich sag mal, wenn allein nur die Deutschen hier leben würden, dann würde es ja irgendwann nur noch alte Menschen hier geben. Also ich verstehe gar nicht, wo da das Problem ist. (weiblich, 16 Jahre, Sozialökologische) 


\section{Kulturelle Bereicherung sehen vor allem die Lebenswelten mit nonkonformistischer Grundhaltung.}

Für die weltoffenen Lebenswelten der Experimentalistischen Hedonisten, Expeditiven und Sozialökologischen ist typisch, dass sie mit der Aufnahme und Integration von Flüchtlingen die Hoffnung auf ein "bunteres Deutschland“ verknüpfen. Mit dem "typisch Deutschen" fangen diese Jugendlichen, die sich nach und nach von den bürgerlichen Konventionen demonstrativ abzugrenzen beginnen, ohnehin wenig an. Bereits in jungen Jahren ist "Multi-Kulti“ als soziale Norm positiv verankert und wird bisweilen auch idealistisch überhöht und romantisiert.

Typische Zitate zur Illustration
( Warum sollte man ihnen kein Asyl gewähren hier in Deutschland, oder warum sollten die nicht hier leben? Ich finde, wenn man irgendwie eine bunte Kultur ist, ist das doch eigentlich viel spannender und viel interessanter zu leben. (männlich, 17 Jahre, Experimentalistische Hedonisten)

( Ich habe damals auch viele Ausländerfreunde gehabt, und man hat denen das auch nicht angesehen auf den ersten Blick so: Du kommst da und da her. Ich finde es auch schön, etwas Neues von denen zu lernen, neue Gerichte, neue Wörter. Das ist dann auch schon interessant. (weiblich, 15 Jahre, Sozialökologische)

( Aber ich glaube, Deutschland ist zum Glück auch so ein bisschen kulturell durchgemischt, dass man nicht so den Stereotyp hat, wo man denkt: Der ist deutsch und alle anderen können nicht deutsch sein. (weiblich, 17 Jahre, Expeditive)

( Ich finde Ausländer eigentlich viel cooler. So viele Schwarze sind halt komplett anders. Ich finde, die sind von der Art her einfach cooler. (weiblich, 15 Jahre, Experimentalistische Hedonisten)

Typisch für diese Gruppen ist auch die Forderung nach einer gerechten und toleranten Gesellschaft ohne Benachteiligung aufgrund von Geschlecht, Religion oder Ethnie. Von den durch das politisch rechte Lager geschürten Klischees distanziert man sich deutlich und fordert, 
dass mehr für den Abbau landläufiger Vorurteile gegen Ausländer getan wird. In diesen Lebenswelten mahnen die Jugendlichen auch entsprechend zu aktivem Widerstand gegen Ausländerfeindlichkeit.

(1) [INT: WÜRDEST DU DICH DENN AUCH SELBST ENGAGIEREN WOLLEN?] $J a$, ich war auch schon auf Demos und sowas. (weiblich, 16 Jahre, Sozialökologische)

Typische Zitate zur Illustration

( Ja, dass halt vor allem diese Unterkünfte eben angegriffen werden, vor allem durch rechtsradikale Leute oder rechtsradikale Parteien (...). Dann sollte man aus sowas dann Konsequenzen ziehen und diese Partei dann auch verbieten. Das hat ja eigentlich nichts mehr mit freier Denkweise zu tun. Das ist ja nur Gewaltanwendung eigentlich. (männlich, 17 Jahre, Experimentalistische Hedonisten)

(7) Die Flüchtlinge stehen in einem falschen Bild da. Bei vielen, vor allem älteren Leuten, stehen die Flüchtlinge wie kriminelle Gammler da, die einfach nur hier schmarotzen wollen, was ja überhaupt nicht stimmt. Und ich finde, die Politik sollte halt gesellschaftlich mal das Bild verändern. Dann würde es vielleicht auch nicht immer solche Brandanschläge geben. Wobei - Nazis lassen sich ja wohl auch nicht vermeiden. (weiblich, 16 Jahre, Sozialökologische)

( Die Vorurteile gegen Ausländer müssten abgebaut werden. Also das sollte alles nicht so verallgemeinert werden: Nur weil mal eine Schlagzeile in der Zeitung steht, dass ein Türke einen Jugendlichen zusammengeschlagen hat, heißt das ja nicht, dass alle Türken so drauf sind. Es gibt aber viele Leute, die so denken. Und das sollte sich ändern. Oder das muss sich ändern. Es ist nicht so, dass in Deutschland nur Deutsche leben und im Ausland nur Ausländer. Es ist halt so, wie es ist. Und da sollten wir offener werden. (männlich, 16 Jahre, Experimentalistische Hedonisten)

Auch in den Gesprächen, die die jugendlichen Interviewer aus der Sozialökologischen und Expeditiven Lebenswelt mit ihren besten Freunden geführt haben, taucht die Frage nach eigenem und staatlichem Engagement für Geflüchtete auf. 
Auszüge aus Fragebögen, die Jugendliche zum Thema „Flüchtlinge“ entwickelt haben.

\section{Flüchtlinge:}

Was fühlst du, wenn in den Medien etwas über Flüchtlinge berichtet wird?

Findest du es wichtig, dass Deutschland Flüchtlinge z.B. aus dem Irak und Syrien aufnimmt?

Sollten in Deutschland/ Europa mehr/weniger Flüchtlinge aufgenommen werden?

Wer sollte bleiben dürfen? Wie kann man das festlegen? Und wer entscheidet das?

Wie würdest du gegen das Flüchtlingśproblem vorgehen?

Was kannst Du selber tun? Was erwartest Du von anderen? (Von wem? Vom Staat?)

weiblich, 17 Jahre, Expeditive

\section{Flücht1 inge}

Wie stehst du zu Flüchtlingen?

$\rightarrow$ Ggf. Konkreter (Flüchtlingsströme, dass wir Flüchtlinge aufnehmen etc.) Engagierst du dich selbst in der Flüchtlingshilfe?

Würdest du gerne?

Wieso?

Was denkst du darüber, dass Flüchtlinge in Turnhallen untergebracht werden? Was hältst du davon, dass Sportunterricht ausfäl1t, weil Turnhallen zu Flüchtlingslagern umfunktioniert werden?

Wie würdest du dich fühlen, wenn DEIN Sportunterricht deswegen ausfäl1t? Wie könnte man deiner Meinung nach Flüchtlinge bzw. Flüchtlingskinder besser integrieren?

weiblich, 17 Jahre, Expeditive

Aktives persönliches Engagement kann man sich nur vereinzelt vorstellen, z. B. im Rahmen von sozialen und schulischen Initiativen. Wichtiger erscheint die Bekämpfung der Fluchtursachen in den Herkunftsländern, die insbesondere von bildungsnahen Jugendlichen thematisiert wird.

( I) habe Verständnis dafür, dass Leute hierherkommen und flüchten. Und ich bin auch keiner, der sagt, dass die jetzt direkt wieder gehen sollen. Keine Ahnung. Im Endeffekt hat das unsere Regierung auch provoziert. Also wenn man die ganze Zeit Waffen dahin liefert, und dann bricht dort ein Krieg aus, sodass es dort keiner mehr aushält und alle 
weg wollen ... Und dann kommen sie halt dahin, wo es schöner ist. (männlich, 16 Jahre, Expeditive)

(Das heißt, man müsste vielleicht echt in den Ländern selbst für Stabilität sorgen, auch wenn ich jetzt nicht so viel zu dem Thema sagen kann, wie man das jetzt ändern könnte. In den nächsten 10 Jahren muss man auf jeden Fall die ganzen Kriege in Syrien, Irak oder in Afrika bekämpfen, die ganzen Bürgerkriege. Dadurch ergibt sich ja auch erst das ganze Flüchtlingsproblem. Ich denke, da muss man auf jeden Fall etwas dagegen tun. (weiblich, 17 Jahre, Expeditive)

\section{Interviewer: weiblich, 17 Jahre, Expeditive}

Es Kommen Ja GERAde ReLATIV VIELE FLÜCHTLINGE NACH DeUtSCHLAND, UND ES WIRD AUCH VIEL IN DEN MEDIEN DARÜBER BERICHTET. WAS FÜHLST DU, WENN DU DARÜBER WAS HÖRST?

Also ich finde es traurig und ich finde, man sollte auch auf jeden Fall was dagegen tun. Nur, ich denke mir manchmal, dass es vielleicht mehr bringen würde, in den Ländern selbst was zu tun, als alle Flüchtlinge aufzunehmen und irgendwo in Flüchtlingslager einzuweisen. Dadurch wird das Problem dort auch nicht gelöst. Und man sollte vielleicht eher an der Basis was ändern, an den Zuständen, die in diesen Flüchtlingslagern herrschen.

ALso Würdest dU, UM GEGEN dAS FLÜCHTLINGSPROBLEM VORZUGEHEN, EHER DIREKT IN DEN LÄNDERN ENTWICKLUNGSHILFE ODER SO WAS LEISTEN?

(Ja, auch. Also klar, man muss sich natürlich in den Ländern, die Flüchtlinge aufnehmen, darauf einstellen. Aber irgendwas sollte man hier natürlich auch ändern, weil du kannst ja auch nicht sagen ok, gut, dann bleibt ihr jetzt auf den ganzen Schiffen, weil das geht ja auch nicht. Aber ich finde es auch wichtig und notwendig, dass man in den Ländern irgendwie Entwicklungshilfe leistet. 
UND DENKST DU, DU KANNST SELBER WAS DAGEGEN TUN, ODER ERWARTEST DU MEHR WAS VON ANDEREN?

( Also ich denke, für Hilfe in den Flüchtlingsländern könnte ich selbst relativ wenig bis gar nichts machen. Ich denke, das ist dann eher auf politischer Basis, dass dort was geregelt werden könnte. Also den Flüchtlingen hier, den könnte man natürlich helfen, wenn man sozialen Projekten beitritt und mithilft, dass die sich hier besser integrieren können. Also da könnte man natürlich schon was machen.

\section{Interviewer: weiblich, 17 Jahre, Sozialökologische}

WAS HÄLTST DU DAVON, DASS VIELLEICHT TEILWEISE AUCH SPORTUNTERRICHT AUSFÄLLT, WEIL EBEN TURNHALLEN ZU FLÜCHTLINGSLAGERN UMFUNKTIONIERT WERDEN?

( Naja, das ist dann eben so. Ich meine es gibt ja keine Alternative. Ich denke mal, dass jede Klasse lieber mal auf zwei Stunden Sport verzichten würde, anstatt mit dem Gewissen zu leben, dass 40 oder 50 Flüchtlinge draußen schlafen mussten.

UND WIE SIEHST DU DAS, WENN DIE TURNHALLEN DANN TOTAL ÜBERFÜLLT SIND?

( Naja, das ist irgendwie so ein Kessel von Krankheiten. Da wird gestohlen, da wird vergewaltigt, da wird verprügelt, da werden Krankheiten weitergegeben, das ist alles nicht so wirklich durchdacht. Vielleicht muss man da mal irgendwie mal wieder Richtlinien schaffen, dass man das mehr kontrolliert. Dass man ärztlich dort irgendwie was stationär hergibt oder so.

KANNST DU NACHVOLLZIEHEN, DASS ES DA TEILWEISE DISKUSSIONEN DARÜBER GIBT, DASS JUGENDLICHE KEINEN SPORTUNTERRICHT HABEN KÖNNEN?

( Ja, na klar. Weil wir helfen! Und dann kommt wieder der Spruch von den Eltern "Wir helfen denen, aber vergessen uns dabei". Aber bei so einem Punkt kann man auch einfach mal kurz ein Auge zudrücken. Das schränkt einen ja auch nicht ein, wenn man jetzt nicht die Turnhalle betreten kann. 


\section{Ja also man könnte Alternativen finden. Siehst du das so?}

(Ja, genau. Dann geht man eben raus. Und macht auf dem Sportplatz oder auf dem Hof dann eine Sporteinheit. Das ist ja nicht so schlimm. Draußen kann man ja Sport machen, man kann eben nur nicht draußen schlafen.

Open Access Dieses Kapitel wird unter der Creative Commons Namensnennung - Nicht kommerziell 2.5 International Lizenz (http://creativecommons.org/licenses/by-nc/2.5/deed.de) veröffentlicht, welche für nicht kommerzielle Zwecke die Nutzung, Verbreitung und Wiedergabe in jeglichem Medium und Format erlaubt, sofern Sie den/die ursprünglichen Autor(en), den Titel des Werks und die Quelle ordnungsgemäß nennen, einen Link zur Creative Commons Lizenz beifügen und im Falle einer Abwandlung durch einen entsprechenden Hinweis deutlich erkennbar machen, dass Änderungen vorgenommen wurden.

Die in diesem Kapitel enthaltenen Bilder und sonstiges Drittmaterial unterliegen ebenfalls der genannten Creative Commons Lizenz, sofern sich aus der Abbildungslegende nichts anderes ergibt. Sofern das betreffende Material nicht unter der genannten Creative Commons Lizenz steht und die betreffende Handlung nicht nach gesetzlichen Vorschriften erlaubt ist, ist auch für die oben aufgeführten nicht-kommerziellen Weiterverwendungen des Materials die Einwilligung des jeweiligen Rechteinhabers einzuholen. 